\title{
Flare stars in nearby Galactic open clusters based on TESS data
}

O. Maryeva ${ }^{1,2}$, K. Bicz ${ }^{3}$, C. Xia ${ }^{4}$, M. Baratella ${ }^{5}$, P. Čechvala ${ }^{6}$ and K. Vida ${ }^{7}$

1 Astronomical Institute of the Czech Academy of Sciences 25165 Ondřejov, The Czech Republic(E-mail: olga.maryeva@asu.cas.cz)

2 Lomonosov Moscow State University, Sternberg Astronomical Institute, Universitetsky pr. 13, 119234, Moscow, Russia

3 Astronomical Institute, University of Wroctaw, Kopernika 11, 51-622 Wroctaw, Poland

4 Department of Theoretical Physics and Astrophysics, Faculty of Science, Masaryk University, Kotlářská 2, 61137 Brno, Czech Republic

5 Dipartimento di Fisica e Astronomia Galileo Galilei, Vicolo Osservatorio 3, 35122, Padova, Italy, (E-mail: martina.baratella.1@phd.unipd.it)

6 Department of Astronomy, Physics of the Earth and Meteorology, Faculty of Mathematics, Physics and Informatics,

Comenius University in Bratislava, Mlynská dolina F-2, 84248 Bratislava, Slovakia

7 Konkoly Observatory, Research Centre for Astronomy and Earth Sciences, H-1121 Budapest, Konkoly Thege Miklós út 15-17, Hungary

Received: October 1, 2020; Accepted: November 11, 2020

\begin{abstract}
The study is devoted to search for flare stars among confirmed members of Galactic open clusters using high-cadence photometry from TESS mission. We analyzed 957 high-cadence light curves of members from 136 open clusters. As a result, 56 flare stars were found, among them 8 hot B-A type objects. Of all flares, $63 \%$ were detected in sample of cool stars $\left(T_{\text {eff }}<5000 \mathrm{~K}\right)$, and $29 \%$ - in stars of spectral type G, while $23 \%$ in K-type stars and approximately $34 \%$ of all detected flares are in M-type stars. Using the FLATW'RM (FLAre deTection With Ransac Method) flare finding algorithm, we estimated parameters of flares and rotation period of detected flare stars. The flare with the largest amplitude appears on the M3 type EQ Cha star. Statistical analysis did not reveal any direct correlation between ages, rotation periods and flaring activity.
\end{abstract}

Key words: Galaxy: open clusters and associations: general - stars: flare stars: activity - space mission: TESS 


\section{Introduction}

Flare stars - stars having detected at least one very short flare in their light curve - are a widely known type of objects in astrophysics. The physical mechanisms leading to the appearance of flares are associated with convective atmospheres, such as in G-M type stars on the main sequence. Thus most of the stars in our Galaxy are potentially flare stars.

In the middle of 20th century, only six flare stars were known (Lippincott, 1952). Since then, a huge progress was made in the field of detecting stellar flares. The interest of astronomers to these objects is associated not only with the incompletely studied mechanisms of formation of flares, but also with the possible influence of flares on habitable zone of potential exoplanets. The Kepler mission had a significant impact on the study of flare stars. As a result,

The Kepler Catalog of Stellar Flares with more than 4000 detected flare stars was made by Davenport (2016). And even more significant impact can be expected from the ongoing Transiting Exoplanet Survey Satellite (TESS) mission, which already covered full celestial sphere. TESS satellite is a space-borne telescope, whose main objective is the detection and study of exoplanets using the technique of transits. However, its database of a high-cadence (2 minute effective sampling) light curves for hundreds of thousands of objects, as well as sequences of Full Frame Images (30 minutes effective sampling), may also provide an invaluable resource for the study of many other kinds of astrophysical objects, including flare stars.

Stellar flares are explosive magnetic reconnection events in a stars magnetosphere (Günther et al., 2020) emitting energy through radio wavelength, X-ray, UV, optical and IR band (Lawson et al., 2019). They show a sharp rise in intensity followed by an exponential decay. Duration of these flares is typically within few minutes to hours (Doyle et al., 2018). The presence of flares indicates magnetic activity in the stellar magnetosphere. Since the magnetic activity is strongly related to the strength of the magnetic field, fast-rotating young stars are typically more active. The magnetic activity also changes over time, so one would expect the frequency and power of flares to change as well. However, it is hard to investigate these changes in G-M type stars due to their long lifetime and slow evolution on the main sequence.

There are several possible ways to estimate the age of such late type stars, such as method based on the rotation period (gyrochronology, Barnes 2007), or spatial motion (kinematic group membership). But probably the most effective and affordable one is by means of estimating an age of a parent cluster. Therefore, the aim of this study is to evaluate the effect of stellar age on the activity of flare stars in Galactic open clusters.

Open clusters are groups of stars loosely held together by their mutual gravitational attraction and formed from the same interstellar molecular cloud. Therefore the age, distance and chemical properties of members in open clusters are approximately identical. This means that the chemical composition of open 
clusters also reflects a real composition of individual members (Netopil et al., 2015).

In this paper, we combine the high temporal resolution light curves acquired by TESS satellite with the cluster membership data based on Gaia DR2 (CantatGaudin et al., 2018a). This allows us to reliably select the members of Galactic open clusters with known age and distance, examine their variability and extract the ones with prominent stellar flares for further statistical analysis.

The paper is organized as follows. In Section 2, we briefly describe a methodology of selection of cluster members having TESS light curves available and the methodology of flare detection and characterisation. In Section 3, we present statistical analysis of detected flares. A conclusion of this study is given in Section 4.

\section{Selection of flare stars}

The first step of our work was the selection of stars belonging to open clusters and being TESS targets with high temporal resolution light curves available. To do it, we took a list of Galactic open clusters from Cantat-Gaudin et al. (2018a) and selected the ones closer than $2 \mathrm{kpc}$ from the Sun. Such distance cut-off allows us to discard the clusters where G0V and later type of dwarfs, the most interesting for flare stars statistics, are below TESS detection limit due to their intrinsic faintness. Then, we used the list of members from Cantat-Gaudin et al. (2018a) for all selected clusters and checked the availability of TESS light curves for every object. As a result, we acquired 859 light curves for stars located in 136 open clusters. We also considered nearby cluster Melote 111 (or Coma Star cluster) with 98 light curves available. Basic parameters of these clusters (age, number of members, distance) are summarized in Table 2, along with the numbers of available light curves and detected flares for them.

As the next step, we performed a visual inspection of all these light curves and discarded the ones without any flares, thus significantly reduced the number of light curves. Then we performed an automated detection and characterisation of the flares using FLATW'RM (FLAre deTection With Ransac Method) code (Vida \& Roettenbacher, 2018). This code uses a machine-learning algorithm to give a robust model of the light curves in order to detect flare events and uses a voting system implemented to keep false positive detections to a minimum. FLATW' RM detects flares and reports the times the flare starts and ends, the time of maximum flux and the maximum percent increase of flux over the light curve around the flare, along with estimated flare energy, either from the raw light curve or by fitting an analytic flare model. It also gives a crude estimation of the period of the underlying light curve, assuming that it is periodic and using the position of the strongest peak in the Lomb-Scargle periodogram.

Figure 1 illustrates the Hertzsprung-Russell (H-R) diagram of several clusters from our analysis with locations of found flare stars and cluster members 
with TESS high temporal resolution light curves available. We also plotted the isochrones calculated using PARSEC (Chen et al., 2014) for metallicities $\mathrm{Z}=0.017$ and $\mathrm{Z}=0.020$ updated to the latest transmission curve calibrated on Gaia DR2 data (Evans et al., 2018) ${ }^{1}$. The figure shows that the majority of the found flare stars have masses between $0.5-2 \mathrm{M}_{\odot}$. Figure 2 presents several clusters where no flare star was detected.
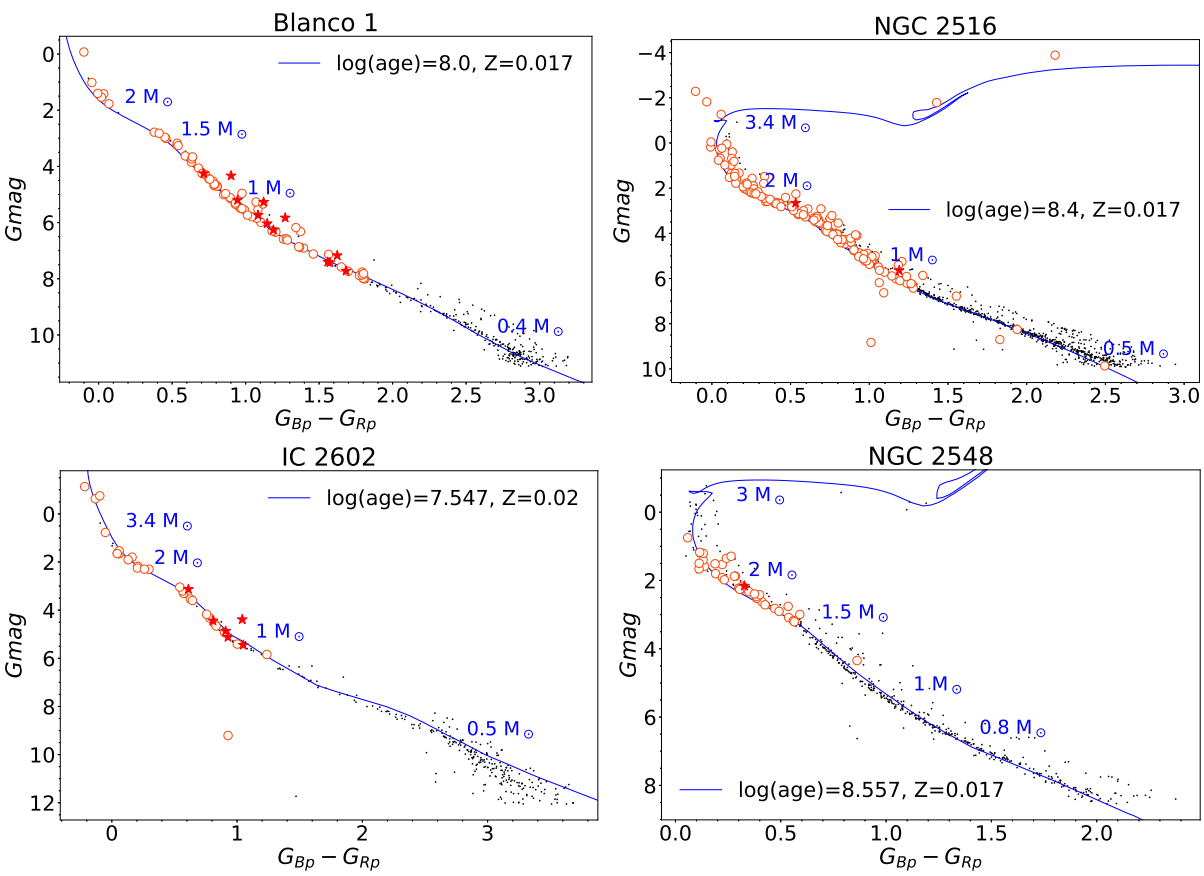

Figure 1. H-R diagram for Blanco 1, NGC 2516, IC 2602 and NGC 2548 clusters based on Gaia photometric data and compared with the PARSEC isochrones (see text for details). Red circles are TESS objects without flares, while red stars - with flares.

It is important to note that two of the clusters - ASCC 19 and Gulliver 6 - are located very close on the sky and also on similar distance. Due to this, the star HD 290527 is considered as a member of both clusters simultaneously (CantatGaudin et al., 2018a). On the other hand, the star HD 290674 is considered to be a member of Gulliver 6 according to Cantat-Gaudin et al. (2018a) and a member of ASCC 19 - according to Cantat-Gaudin et al. (2018b). This leads to the ambiguities of the ages for these stars.

${ }^{1}$ PARSEC isochrones in Gaia DR2 passbands are available at http://stev.oapd.inaf.it/ cgi-bin/cmd 

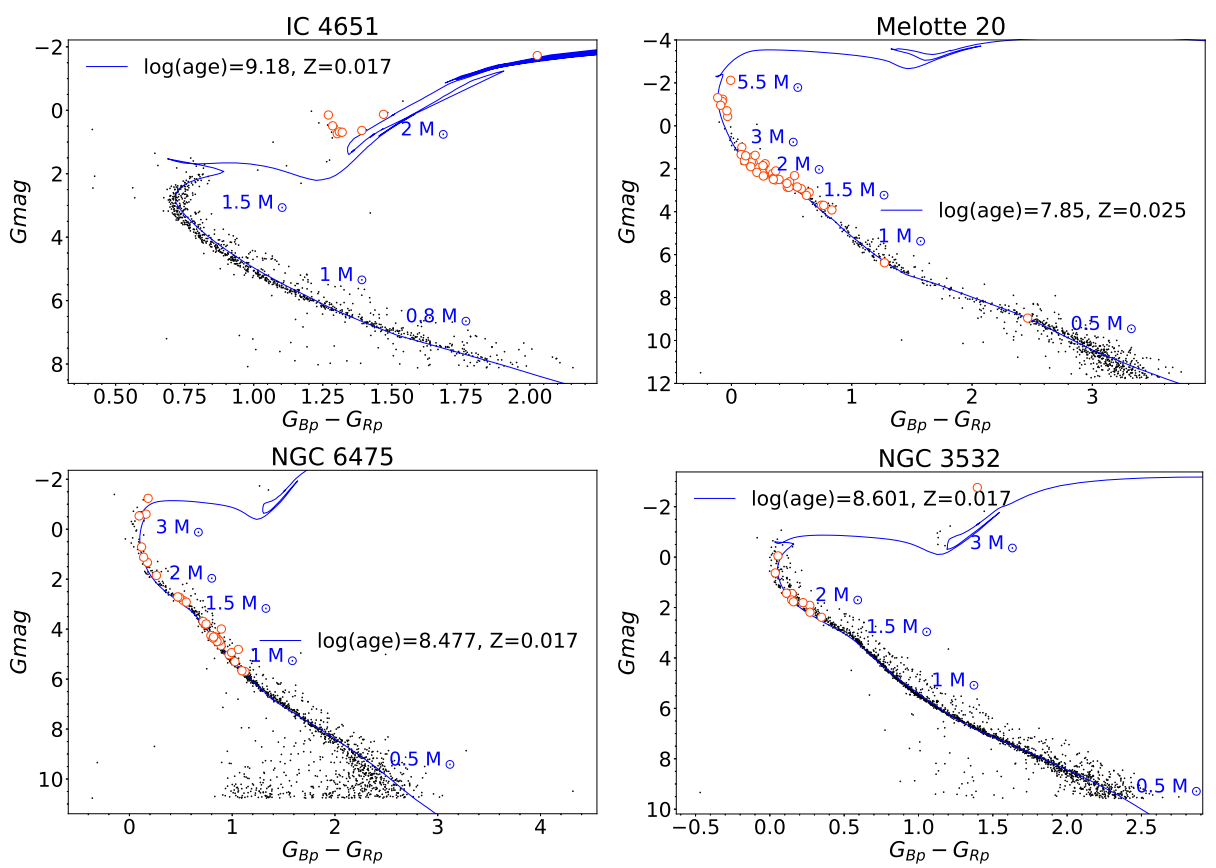

Figure 2. H-R diagram for IC 4651, Melotte 20, NGC 6475 and NGC 3532 clusters based on Gaia photometric data and compared with PARSEC isochrones. Red circles are TESS objects without flares.

A full list of flare stars we found is given in Table 1. Specified spectral types are taken from the SIMBAD database. As well as spectral types are not known for all star, 5th column gives effective temperatures $T_{\text {eff }}$ according to Gaia DR2 (Gaia Collaboration et al., 2016, 2018). The 6th column contains the number of flares detected during the period of observations, while the last column is the period of rotation. Both values were estimated using FLATW'RM. The code estimates the periods based on the strongest peak in the Lomb-Scargle periodogram, and thus may provide bogus or halved periods in some cases. Therefore we also checked all measured period values by eye and corrected them where necessary so that they clearly correspond to the main periodic components. For one of the stars in our sample - EO Cha - the determined period is close to total duration of observations and might be caused by systematic effects in the data. However, our value is in agreement with the one reported in AAVSO VSX database ${ }^{2}$, and thus we decided to keep it in our analysis.

${ }^{2}$ American Association of Variable Star Observers Variable Star Index is available in https : //www . aavso.org/vsx/ 
Table 1. List of flare stars selected in this work. Spectral types are taken from the SIMBAD database. $T_{\text {eff }}$ is temperature taken from Gaia DR2 (Gaia Collaboration et al., 2016, 2018). Numbers of flares $\left(N_{\mathrm{fl}}\right)$ and rotation periods $\left(P_{\mathrm{rot}}\right)$ are estimated as described in Section 2.

\begin{tabular}{|c|c|c|c|c|c|c|}
\hline Cluster & Gaia DR2 ID & Name & $\begin{array}{l}\text { Sp. } \\
\text { type }\end{array}$ & $T_{\text {eff }}$ & $N_{\mathrm{fl}}$ & $\begin{array}{c}P_{\text {rot }} \\
{[\text { days }]}\end{array}$ \\
\hline $\operatorname{ASCC} 19^{a}$ & 3220225706694228096 & HD 290527 & A3 & 7470 & 3 & 6.69 \\
\hline ASCC 21 & 3236083864117227264 & HD 36030 & B9V & 9777 & 1 & - \\
\hline Alessi 13 & 4861032719915154176 & - & - & 3922 & 1 & 2.27 \\
\hline Alessi 13 & 4860643905115917312 & CD-36 1309 & F8 & 5922 & 4 & 1.20 \\
\hline Alessi 13 & 4854771001195018752 & CD-37 1263 & G5 & 5637 & 5 & 2.06 \\
\hline Blanco 1 & 2320842551136015872 & & $\mathrm{~K} 4-5 \mathrm{~V}$ & 4954 & 1 & 5.55 \\
\hline Blanco 1 & 2320838943363481984 & - & K5.3 & 4296 & 5 & 2.17 \\
\hline Blanco 1 & 2320874054720005248 & - & $\mathrm{F}-\mathrm{K}$ & 4131 & 1 & 5.84 \\
\hline Blanco 1 & 2320933157765826560 & CD-30 19826 & F-K & 5496 & 3 & 1.03 \\
\hline Blanco 1 & 2320596123092361088 & - & F-K & 5031 & 2 & 0.34 \\
\hline Blanco 1 & 2320862200611102848 & - & F-K & 5499 & 7 & 2.34 \\
\hline Blanco 1 & 2320860074602561408 & - & F-K & 4987 & 2 & 0.41 \\
\hline Blanco 1 & 2320881377640416640 & - & F-K & 4301 & 3 & 1.18 \\
\hline Blanco 1 & 2320869897192498432 & CD-30 19800 & F8 & 6070 & 1 & 2.65 \\
\hline Blanco 1 & 2320795031617857152 & & F-K & 4977 & 3 & 3.15 \\
\hline Blanco 1 & 2320872650266808192 & - & F-K & 4873 & 3 & 4.14 \\
\hline Blanco 1 & 2320847533296914560 & - & F-K & 4579 & 4 & 6.47 \\
\hline Gulliver 6 & 3217389348947353728 & HD 290674 & A0 & 9034 & 1 & 6.41 \\
\hline IC 2602 & 5239841340702856960 & HD 93405 & $\mathrm{~F} 3 / 5 \mathrm{~V}$ & 6383 & 5 & 0.75 \\
\hline IC 2602 & 5239660372284736896 & - & - & 5362 & 4 & 3.80 \\
\hline IC 2602 & 5239626420542800512 & V570 Car & G9Ve & 5071 & 2 & 1.21 \\
\hline IC 2602 & 5239498744077038976 & HD 310131 & G6V & 5583 & 2 & 0.62 \\
\hline IC 2602 & 5253546997989686912 & HD 307772 & G7Ve & 5161 & 3 & 0.32 \\
\hline IC 2602 & 5251470948229949568 & - & - & 5768 & 1 & 3.02 \\
\hline Mamajek 1 & 5209082129256747008 & EG Cha & $\mathrm{K} 4 \mathrm{Ve}$ & 4365 & 8 & 4.35 \\
\hline Mamajek 1 & 5209135352491538432 & EO Cha & M0 & 4089 & 3 & 22.26 \\
\hline Mamajek 1 & 5209038423669475712 & EL Cha & M2 & 3526 & 6 & 1.84 \\
\hline Mamajek 1 & 5215178848217868288 & - & M3 & 3673 & 2 & 2.96 \\
\hline Mamajek 1 & 5209118305765620096 & EQ Cha & M3 & 3367 & 14 & 1.25 \\
\hline NGC 1662 & 3294740954034417792 & - & - & 4454 & 1 & - \\
\hline NGC 1662 & 3295557822453425792 & - & - & 4447 & 1 & - \\
\hline NGC 2422 & 3030122727536859008 & - & - & 4826 & 1 & 6.97 \\
\hline NGC 2451A & 5538425822158520576 & - & - & 3761 & 2 & 0.52 \\
\hline
\end{tabular}


Table 1. Continued.

\begin{tabular}{ccccccc}
\hline Cluster & Gaia DR2 ID & Name & $\begin{array}{c}\text { Sp. } \\
\text { type }\end{array}$ & $T_{\text {eff }}$ & $N_{\text {fl }}$ & $\begin{array}{c}P_{\text {rot }} \\
\text { [days] }\end{array}$ \\
\hline NGC 2451B & 5538722449779987072 & - & - & 4438 & 1 & 0.50 \\
NGC 2451B & 5538818176010943488 & - & - & 5326 & 5 & 1.95 \\
NGC 2516 & 5290732721731067136 & - & - & 4882 & 2 & 2.07 \\
NGC 2516 & 5290719115275116288 & - & A7III & 7038 & 1 & 1.02 \\
NGC 2548 & 3064487035741401984 & - & A2/4 & 8160 & 1 & 0.80 \\
NGC 6281 & 5976330585929049600 & V948 Sco & ApSi & 8553 & 1 & 1.64 \\
Platais 8 & 5305106426785000448 & CPD-55 1885 & G5V & 5511 & 3 & 0.92 \\
Platais 8 & 5303854293507991680 & HD 78027 & A1V & 8521 & 1 & 3.31 \\
Platais 8 & 5310127655876188544 & CD-55 2543 & G8V & 5406 & 1 & 3.84 \\
Platais9 & 5427272240335502848 & - & - & 3316 & 1 & - \\
Platais9 & 5427469843189439360 & - & - & 3759 & 1 & 3.49 \\
Platais9 & 5423893028786327168 & HD 80484 & A1V & 8884 & 1 & 0.69 \\
Platais9 & 5327362504934951040 & & - & 5732 & 1 & 1.23 \\
Melotte 111 & 3959841756787187456 & - & M3.0 & 3395 & 2 & - \\
Melotte 111 & 4002505586787874560 & - & M3.5 & 3421 & 5 & - \\
Melotte 111 & 3960130997064687616 & - & M3.5 & 3783 & 5 & - \\
Melotte 111 & 4008440617411511552 & Sand 64 & M3.9 & 3921 & 2 & - \\
Melotte 111 & 4009049575054518400 & - & M2.4 & 3789 & 1 & - \\
Melotte 111 & 3954074577781239936 & Sand 63 & M2.2 & 3628 & 2 & - \\
Melotte 111 & 4009398051520907008 & HK Com & M4.2 & 3848 & 4 & - \\
Melotte 111 & 4009440382718550272 & - & M3.7 & 3937 & 1 & - \\
Melotte 111 & 4009455054326835968 & - & M2.6 & 3899 & 3 & 3.39 \\
Melotte 111 & 4002543008838439296 & - & M3.8 & - & 1 & 1.87 \\
\hline \hline
\end{tabular}

${ }^{a}$ HD 290527 is probably a member of Gulliver 6 cluster, see text for details.

\section{Discussion}

\subsection{Spectral distribution of selected flare stars}

Most of stellar flares occur in red dwarfs, more often in cool M dwarfs (Güdel \& Naz, 2009). Data from the first data release of TESS mission confirm it: among 1228 flare stars detected by Günther et al. (2020), 673 objects were classified as M dwarfs. Our data is in a good agreement with these previous studies. Figure 3 presents the histogram of the number of flare stars versus spectral type based on the data from Table 1. Indeed, the majority of selected objects are stars of late spectral type. 
M-type dwarfs are very faint objects, an absolute visual magnitude of M0V star is $M_{V}=9.0 \mathrm{mag}$ and for M5V is $M_{V}=14.6 \mathrm{mag}$ (Schmidt-Kaler, 1982). In our work, we found M-type flare stars only in two nearby open clusters, Mamajek 1 (distance $D=98.72 \mathrm{pc}$ ) and Mel $111(D=96 \mathrm{pc}$ ). Figure 4 shows that flares were detected for almost $50 \%$ of all considered M-type stars.

Stellar flares have been previously detected in some hot B-A type stars (in optical range (Schaefer, 1989; Balona, 2012); in X-ray (Schmitt et al., 1994; Yanagida et al., 2007)). Several objects from our sample are also early spectral type flare stars. In this study, we detected flares in seven A-type stars (Table 1). One of them - V948 Sco - belongs to $\alpha^{2}$ CVn variables, chemically peculiar main sequence stars with a strong magnetic field. For all other A-type stars, the variability was registered for the first time.
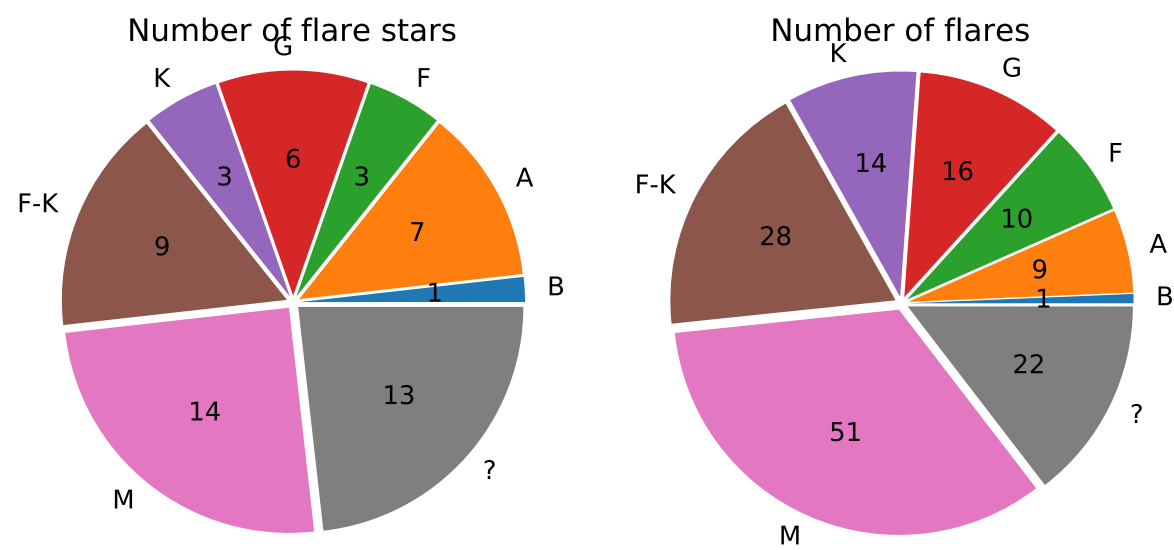

Figure 3. Distribution of flare stars in our sample (left panel), and detected flares from them (right panel) over spectral types from Table 1.

Another selected hot star is HD 36030, classified as B9V star by Houk \& Swift (1999). Figure 5 presents the light curve of HD 36030 and a clearly detected flare. As in the case with A-type stars, we did not find any previously published work about variability of this object.

Flares of hot stars may be explained through binarity. As mostly A-type stars are probably double or multiple systems, it is natural to expect flares to originate in a cool companion. However, Balona (2019) gives strong arguments that flares originate in A-type stars themselves. These arguments are based on ratio of luminosity: an M- or K-type companion is about 50-100 times less luminous than an A-type star. The flare amplitude originating in a cool companion should therefore, on average, appears to be about 50-100 times smaller when observed together with A-type star. However, flares detected in hot stars are comparable with their luminosity (Balona, 2019). Flares in A-stars of our sam- 

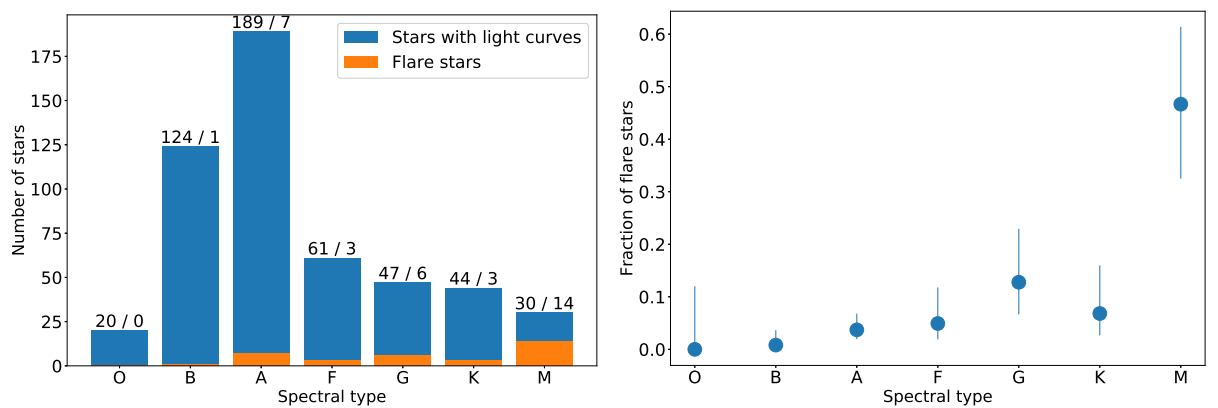

Figure 4. Left panel - histogram of total number of light curves and flare stars among them per spectral type (where it was reliably determined).

Right panel - fraction of flare stars as a function of spectral type. Error bars depict the $90 \%$ confidence intervals for the corresponding values.
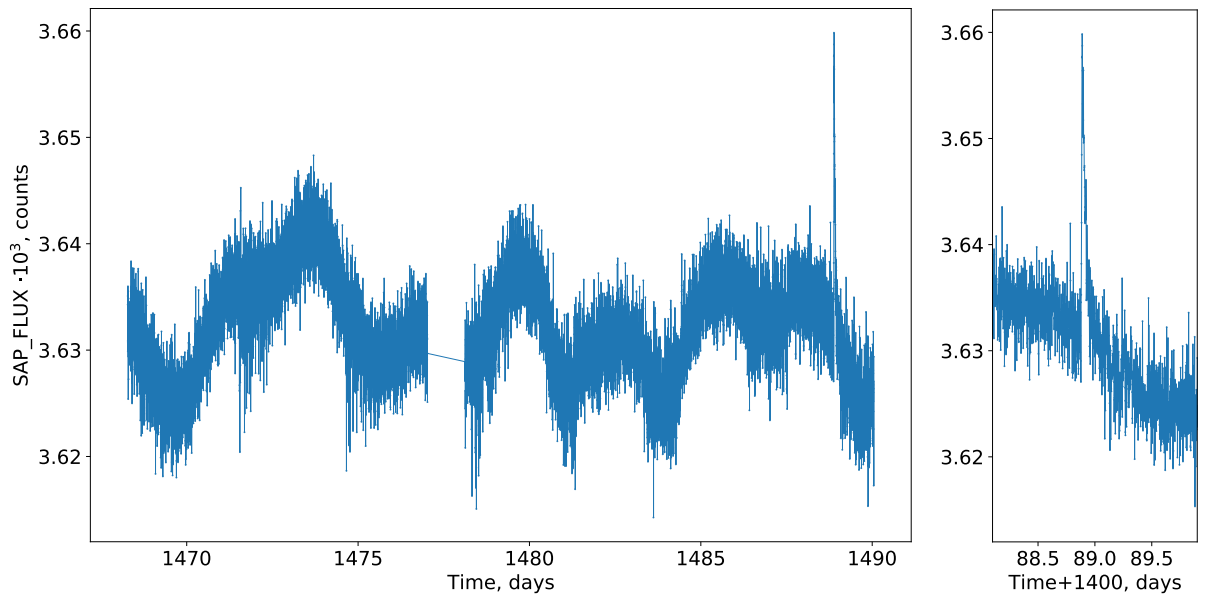

Figure 5. Light curve of HD 36030 (B9V) with a clearly detected flare.

ple have amplitudes of couple percents only, and may be in principle, at least partially, explained by a giant flares with amplitudes of several magnitudes on a late type companion, like the ones reported in Beskin et al. (2017). However, such giant flares are quite rare, and are not detected in our sample of M stars. Thus, we may not expect them to be abundant among the possible components of our A stars too. On the other hand, magnetic interaction with the companion star could provide a plausible explanation for observed flaring activity (Balona, 2012).

According to Balona (2015), the relative number of flare stars from cool M dwarfs to hot A-type stars is probably the same. According to our results (see 
Figure 4), the fraction of flare stars among G-M type objects is significantly higher.

There are already no published data about spectral types for fourteen flare stars in our sample (see Table 1, Figure 3). Future spectral observations of these objects could improve statistics.

\subsection{Statistical analysis of flares}

The mean duration of flares for stars in Table 1 varies between 20 to 70 minutes. This timescale is comparable with the one of GOES X-class solar flares, quite often visible in the white light (Harra et al., 2016). Only 4 stars among 56 analysed in this paper have more than 5 flares during period of TESS observations. One of them is EQ Cha (M3) - variable star of Orion type, close visual binary (Sicilia-Aguilar et al., 2009) and a member of Mamajek 1 open cluster. It showed 14 flares during 25 days of observations. Flaring activity of EQ Cha star has been already reported in X-ray by López-Santiago et al. (2010).
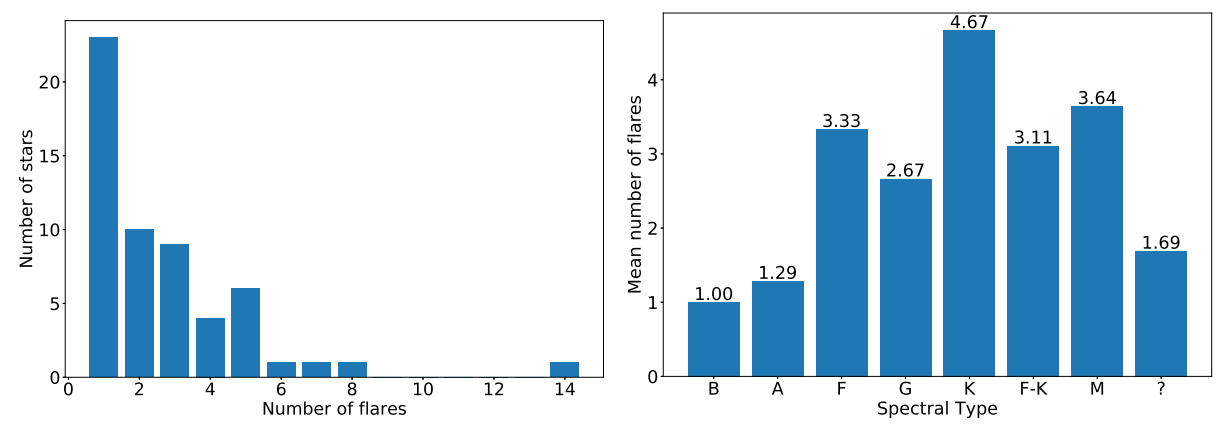

Figure 6. Histogram of number of flares detected from a star (left panel). Mean number of flares per star depending on spectral class from Table 1 (right panel).

On the right panel of Figure 6, we can see that K-type stars have the largest mean flare activity in their light curves. The largest number of flares belongs to M-type stars, which are all earlier than M4 in our sample (see Table 1) and thus are cool stars with convective envelope, as well as to K-type stars. These $\mathrm{K}$ and early $\mathrm{M}$ dwarfs, which retain a radiative core, presumably all have a quite efficient solar-type dynamo powering their magnetic activity. On the other hand, M dwarfs of spectral type later than M4 are fully convective, and have a turbulent dynamo at work (Wright et al., 2011; Stassun et al., 2011). The activity of G-type stars could be also associated with their convective envelopes. Figure 7 shows the dependence of a flare rate on the star' effective temperature.

The appearance of spots on the surface of stars and stellar flares are clear evidences of stellar magnetism. The connection between stellar rotation and magnetic activity has been studied in many works. For example, Guo et al. 


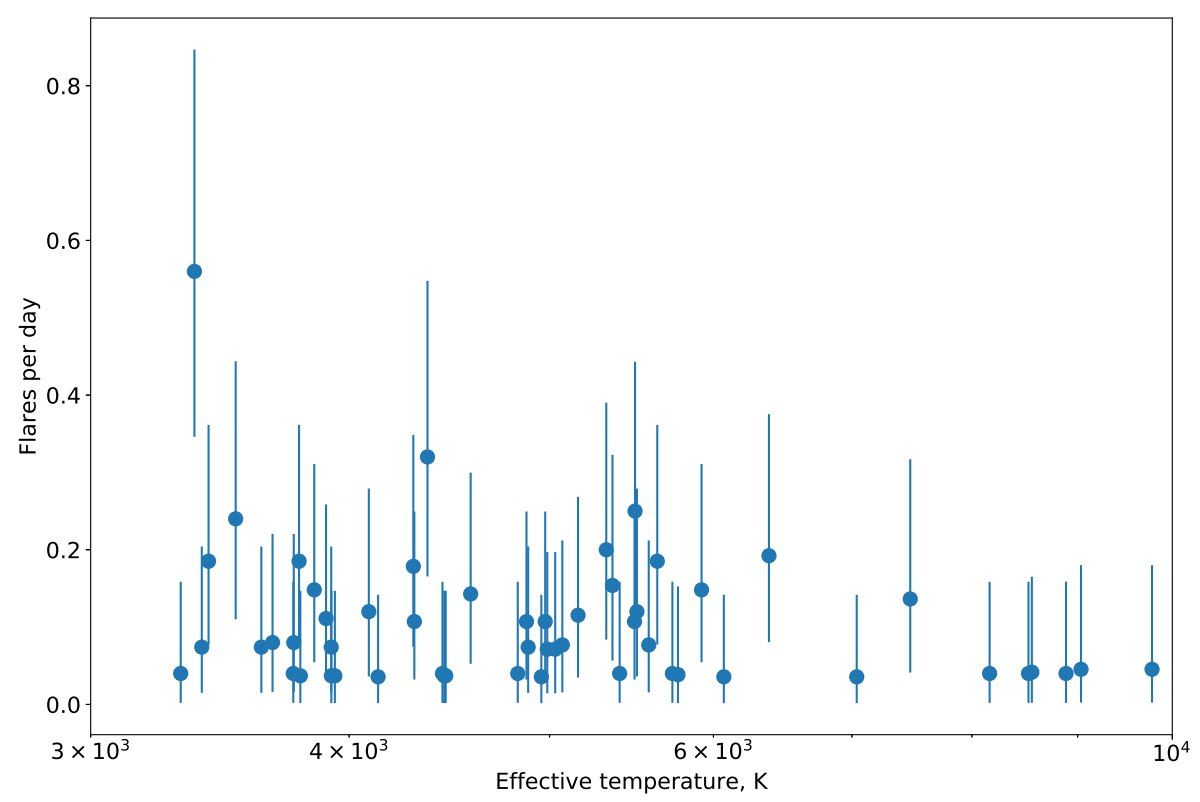

Figure 7. Rate of stellar flares as a function of temperature. Error bars correspond to the $90 \%$ confidence intervals computed assuming Poissonian statistics.

(2014) analyzed X-class flares of Sun during the 22nd and 23rd solar cycles, and they found that flares closely follow the same 11 year cycle as sunspots. Doyle et al. (2018) did not find any correlation between the rotation phase and the number of flares for M-dwarfs based on Kepler data, but clearly detected the decrease of flaring activity for rotational periods longer than 10 days. The same dependence is seen in X-ray activity-rotation relation (Pizzocaro et al., 2019).

Figure 8 shows the dependence of flaring activity in our sample, estimated as a number of flares per day of observations on the rotational period of the star. We may spot just a slight trend of decreasing activity towards slower rotation. While we may not stress it as a significant dependence, let's note that it agrees with the expected behaviour of magnetic activity being as a result of internal magnetic dynamo, arising from the combination of stellar differential rotation and convection in the sub-photospheric layers.

\subsection{Age distribution of flare stars}

Mamajek 1 is the youngest open cluster among ones where we have found flare stars. Its age is $6 \mathrm{Myr}$. The oldest one where we have found flare is the cluster NGC 1662. Its age 1 Gyr is comparable with the maximal lifetime of open clusters (Fujii \& Portegies Zwart, 2016). The ages of all examined clusters span from 1 Myr (IC 5146, the youngest) to 4.3 Gyr (NGC 188, the oldest one). Therefore, 


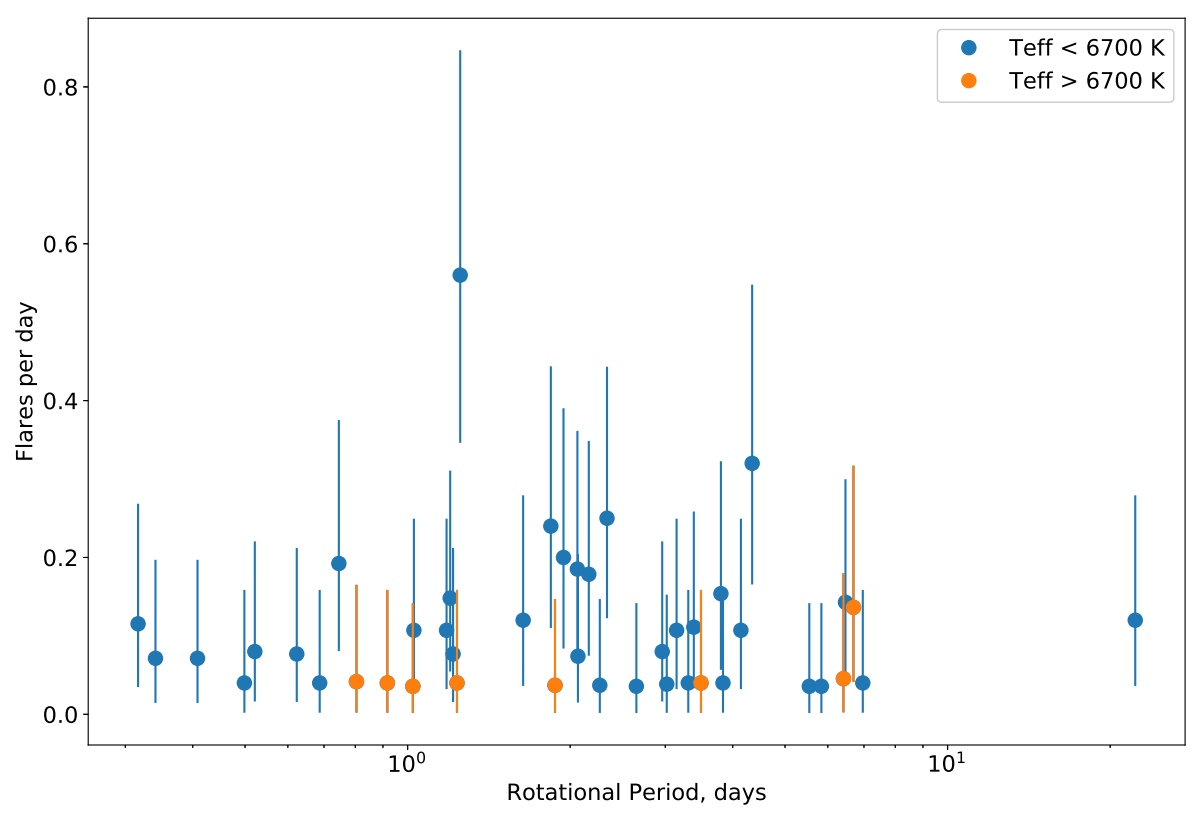

Figure 8. Rate of stellar flares as a function of rotational period. Error bars correspond to the $90 \%$ confidence intervals computed assuming Poissonian statistics.

the collected data do not allow us to confidently determine the age after which flare stars in clusters disappear.

Figure 9 shows how flaring activity changes with stellar age. The resulting distribution is mostly flat, with just a single deviating point with 14 detected flares from EQ Cha star. We therefore cannot reliably see any dependence of flaring activity on the age.

\section{Conclusion}

In order to study the dependence of stellar flaring activity on the age, we performed an uniform study of large sample of Galactic open clusters from catalogue of Cantat-Gaudin et al. (2018a). We analyzed a total amount of 957 high-cadence TESS light curves for stars from 136 open clusters. By visual inspection and later using a FLATW'RM code, it finally turned out that 56 of them are flare stars with 151 flares detected.

$41.5 \%$ of all flares were detected in cool stars. 8 flare stars belong to Band A-type stars. The flare activity was detected for the first time for all these hot stars. The flare with the largest amplitude appears on M3-type EQ Cha star. From total amount of detected flare stars in our sample, $25 \%$ of them 


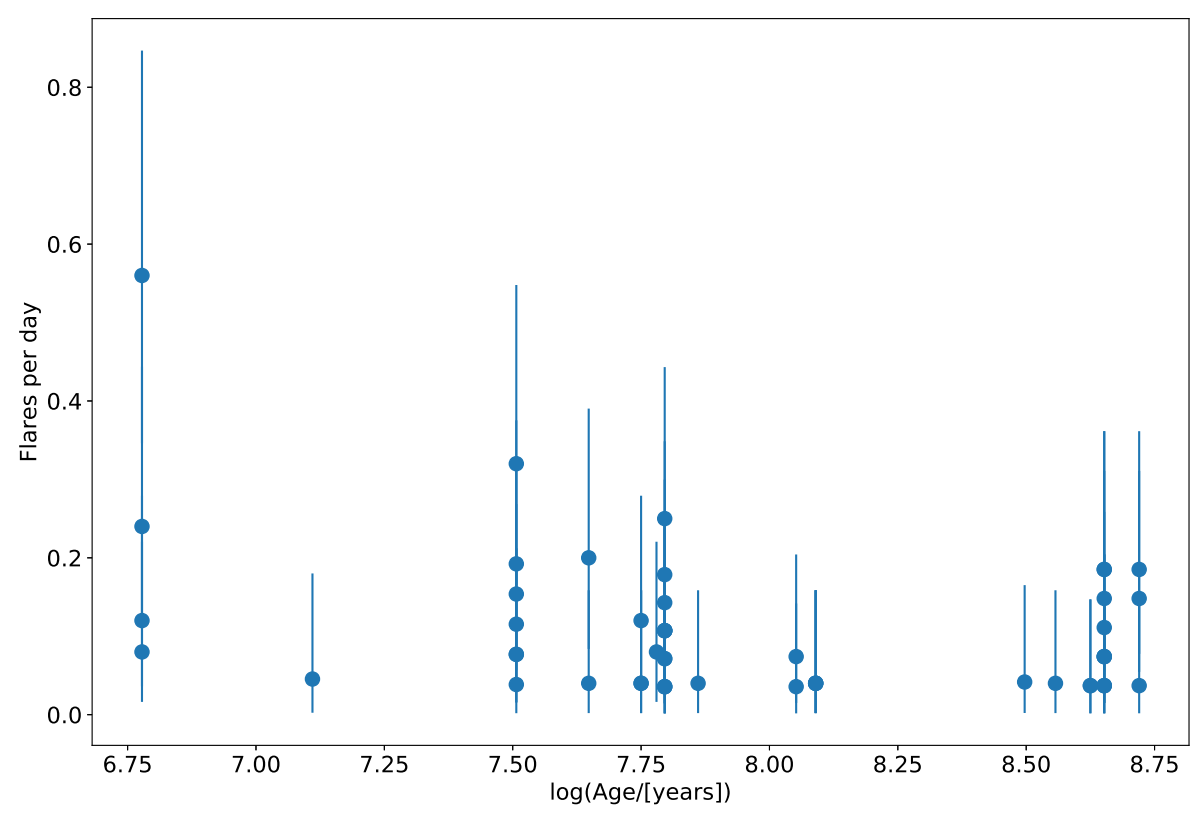

Figure 9. Rate of flares as a function of age. The rate was estimated from the number of detected flares divided by the effective light curve duration for every individual star. Error bars depict the $90 \%$ confidence intervals computed assuming Poissonian statistics.

are members of spectral type M, $11 \%$ are G-type stars and about 5.5\% F- and K-type stars.

Statistical analysis of detected flare stars shows that:

- among cool stars up to $50 \%$ have flares, while among A-type stars it is only $3.7 \%$.

- duration of flares varies between 20 to 70 minutes.

- we found no significant link between rotation rate and flare activity.

- we found no significant link between stellar age and flare activity.

In the study, we used TESS data recorded at 2-minute cadence light curves, which are an invaluable source of photometric data for various kinds of stars, pre-selected before the mission start. Although TESS full-frame images have a much wider coverage of objects, limited only by crowding, their utility for studies of stellar flares are significantly lower due to much worse temporal resolution (30 minutes, which is comparable to total duration of flares). As TESS mission now continues into its third year, re-visiting the regions of the sky already observed 
before, we expect to get more data for the objects we studied in the future. In the future, we plan to do spectroscopic observations of B-A type stars with flare activity we had detected, in order to investigate their possible multiplicity.

Acknowledgements. The authors are extremely thankful to organizers of GATE Summer School for the idea of this project. The authors acknowledge the support from ERASMUS+ grant number 2017-1-CZ01-KA203-035562. We are grateful to the anonymous referee for useful comments and suggestions on the manuscript. O.M. acknowledges support from the Czech Science Foundation GA18-05665S. K.V. acknowledges the support of the Lendület Program of the Hungarian Academy of Sciences, project No. LP2018-7/2019, the NKFI KH-130526 and NKFI K-131508 grants.

This paper includes data collected by the TESS mission, which are publicly available from the MikulskiArchive for Space Telescopes (MAST). Funding for the TESS mission is provided by NASA's Science Mission directorate. This research was made by using of the SIMBAD data base and the VizieR catalogue access tool, both operated at CDS, Strasbourg, France. The WEBDA database, operated at the Department of Theoretical Physics and Astrophysics of the Masaryk University, and data from the European Space Agency (ESA) mission Gaia $^{3}$, processed by the Gaia Data Processing and Analysis Consortium $\left(\mathrm{DPAC}^{4}\right)$. Funding for the DPAC has been provided by national institutions, in particular the institutions participating in the Gaia Multilateral Agreement.

\section{References}

Balona, L. A., Kepler observations of flaring in A-F type stars. 2012, Monthly Notices of the RAS, 423, 3420, DOI: 10.1111/j.1365-2966.2012.21135.x

Balona, L. A., Flare stars across the H-R diagram. 2015, Monthly Notices of the RAS, 447, 2714, DOI: 10.1093/mnras/stu2651

Balona, L. A., Pulsation, Rotation and Flares in A Stars. 2019, in IAU Symposium, Vol. 339, Southern Horizons in Time-Domain Astronomy, ed. R. E. Griffin, 77-82

Barnes, S. A., Ages for illustrative field stars using gyrochronology: viability, limitations and errors. 2007, Astrophysical Journal, 669, 1167, DOI: 10.1086/519295

Beskin, G., Karpov, S., Plokhotnichenko, V., Stepanov, A., \& Tsap, Y., Polarimetric Observations of Flare Stars. 2017, in Astronomical Society of the Pacific Conference Series, Vol. 510, Stars: From Collapse to Collapse, ed. Y. Y. Balega, D. O. Kudryavtsev, I. I. Romanyuk, \& I. A. Yakunin, 303

Bossini, D., Vallenari, A., Bragaglia, A., et al., Age determination for 269 Gaia DR2 open clusters. 2019, Astronomy and Astrophysics, 623, A108, DOI: 10.1051/0004$6361 / 201834693$

Cantat-Gaudin, T., Jordi, C., Vallenari, A., et al., A Gaia DR2 view of the open cluster population in the Milky Way. 2018a, Astronomy and Astrophysics, 618, A93, DOI: 10.1051/0004-6361/201833476

\footnotetext{
${ }^{3}$ https://www.cosmos.esa.int/gaia

${ }^{4}$ https://www.cosmos.esa.int/web/gaia/dpac/consortium
} 
Cantat-Gaudin, T., Vallenari, A., Sordo, R., et al., Characterising open clusters in the solar neighbourhood with the Tycho-Gaia Astrometric Solution. 2018b, Astronomy and Astrophysics, 615, A49, DOI: 10.1051/0004-6361/201731251

Chen, Y., Girardi, L., Bressan, A., et al., Improving PARSEC models for very low mass stars. 2014, Monthly Notices of the RAS, 444, 2525, DOI: 10.1093/mnras/stu1605

Davenport, J. R. A., The Kepler Catalog of Stellar Flares. 2016, Astrophysical Journal, 829, 23, DOI: $10.3847 / 0004-637 \mathrm{X} / 829 / 1 / 23$

Doyle, L., Ramsay, G., Doyle, J. G., Wu, K., \& Scullion, E., Investigating the rotational phase of stellar flares on M dwarfs using K2 short cadence data. 2018, Monthly Notices of the RAS, 480, 2153, DOI: 10.1093/mnras/sty1963

Evans, D. W., Riello, M., De Angeli, F., et al., Gaia Data Release 2. Photometric content and validation. 2018, Astronomy and Astrophysics, 616, A4, DOI: 10.1051/0004-6361/201832756

Fujii, M. S. \& Portegies Zwart, S., The Formation and Dynamical Evolution of Young Star Clusters. 2016, Astrophysical Journal, 817, 4, DOI: 10.3847/0004$637 \mathrm{X} / 817 / 1 / 4$

Gaia Collaboration, Brown, A. G. A., Vallenari, A., et al., Gaia Data Release 2. Summary of the contents and survey properties. 2018, Astronomy and Astrophysics, 616, A1, DOI: 10.1051/0004-6361/201833051

Gaia Collaboration, Prusti, T., de Bruijne, J. H. J., et al., The Gaia mission. 2016, Astronomy and Astrophysics, 595, A1, DOI: 10.1051/0004-6361/201629272

Güdel, M. \& Naz, Y., X-ray spectroscopy of stars. 2009, The Astronomy and Astrophysics Review, 447, 309, DOI: 10.1007/s00159-009-0022-4

Günther, M. N., Zhan, Z., Seager, S., et al., Stellar Flares from the First TESS Data Release: Exploring a New Sample of M Dwarfs. 2020, Astronomical Journal, 159, 60, DOI: $10.3847 / 1538-3881 / \mathrm{ab} 5 \mathrm{~d} 3 \mathrm{a}$

Guo, J., Lin, J., \& Deng, Y., The dependence of flares on the magnetic classification of the source regions in solar cycles 22-23. 2014, Monthly Notices of the RAS, 441, 2208, DOI: $10.1093 /$ mnras/stu695

Harra, L. K., Schrijver, C. J., Janvier, M., et al., The Characteristics of Solar X-Class Flares and CMEs: A Paradigm for Stellar Superflares and Eruptions? 2016, Solar Physics, 291, 1761, DOI: 10.1007/s11207-016-0923-0

Houk, N. \& Swift, C., Michigan catalogue of two-dimensional spectral types for the HD Stars, Vol. 5. 1999, Michigan Spectral Survey, 5, 0

Lawson, K. D., Wisniewski, J. P., Bellm, E. C., Kowalski, A. F., \& Shupe, D. L., Identification of Stellar Flares Using Differential Evolution Template Optimization. 2019, Astronomical Journal, 158, 119, DOI: 10.3847/1538-3881/ab3461

Lippincott, S. L., Search for flares of dM stars on the sproul astrometric programm . 1952, Astrophysical Journal, 115, 582, DOI: 10.1086/145584

López-Santiago, J., Albacete Colombo, J. F., \& López-García, M. A., Deep XMMNewton observation of the $\eta$ Chamaleontis cluster. 2010, Astronomy and Astrophysics, 524, A97, DOI: 10.1051/0004-6361/201015306 
Netopil, M., Paunzen, E., \& Cararro, G., A comparative study on the reliability of open cluster parameters. 2015, Astronomy and Astrophysics, 582, 19, DOI: 10.1051/0004$6361 / 201526372$

Pizzocaro, D., Stelzer, B., Poretti, E., et al., Activity and rotation of the X-ray emitting Kepler stars. 2019, Astronomy and Astrophysics, 628, A41, DOI: 10.1051/0004$6361 / 201731674$

Röser, S., Schilbach, E., \& Goldman, B., Nine new open clusters within 500 pc from the Sun. 2016, Astronomy and Astrophysics, 595, A22, DOI: 10.1051/0004$6361 / 201629158$

Schaefer, B. E., Flashes from Normal Stars. 1989, Astrophysical Journal, 337, 927, DOI: $10.1086 / 167162$

Schmidt-Kaler, T. 1982, Stars and Star Clusters, ed. K. Schaifers \& H. H. Voigt

Schmitt, J. H. M. M., Guedel, M., \& Predehl, P., Spatially resolved X-ray and radio observations of Castor A+B+C. 1994, Astronomy and Astrophysics, 287, 843

Sicilia-Aguilar, A., Bouwman, J., Juhász, A., et al., The Long-Lived Disks in the $\eta$ Chamaeleontis Cluster. 2009, Astrophysical Journal, 701, 1188, DOI: 10.1088/0004$637 \mathrm{X} / 701 / 2 / 1188$

Stassun, K. G., Hebb, L., Covey, K., et al., The M4 Transition: Toward a Comprehensive Understanding of the Transition into the Fully Convective Regime. 2011, in Astronomical Society of the Pacific Conference Series, Vol. 448, 16th Cambridge Workshop on Cool Stars, Stellar Systems, and the Sun, ed. C. Johns-Krull, M. K. Browning, \& A. A. West, 505

Vida, K. \& Roettenbacher, R. M., Finding flares in Kepler data using machine-learning tools. 2018, Astronomy and Astrophysics, 616, 163, DOI: https://doi.org/10.1051/0004-6361/201833194

Wright, N. J., Drake, J. J., Mamajek, E. E., \& Henry, G. W., The Stellar-activityRotation Relationship and the Evolution of Stellar Dynamos. 2011, Astrophysical Journal, 743, 48, DOI: 10.1088/0004-637X/743/1/48

Yanagida, T., Ezoe, Y., Kawaharada, M., Kokubun, M., \& Makishima, K., Large Xray Flares from B-Type Stars, HD261902 and HD47777, in NGC2264 Observed with CHANDRA. 2007, Astronomical Society of the Pacific Conference Series, 361, 533

Yen, S. X., Reffert, S., Schilbach, E., et al., Reanalysis of nearby open clusters using Gaia DR1/TGAS and HSOY. 2018, Astronomy and Astrophysics, 615, A12, DOI: 10.1051/0004-6361/201731905 


\section{A. Clusters with TESS light curves available}

Table 2. Complete list of Galactic open clusters investigated in this work, with ages $\tau$ taken from various sources (see the last column), distances $D$ and number of members $N_{\text {mem }}$ taken from Cantat-Gaudin et al. (2018a), number of available light curves $N_{\mathrm{lc}}$ in the TESS database and number of flare stars $N_{\mathrm{fs}}$ estimated in this work.

\begin{tabular}{lcccccr}
\hline \hline Name & $\log (\tau /[\mathrm{yr}])$ & $D[\mathrm{pc}]$ & $N_{\text {mem }}$ & $N_{\mathrm{lc}}$ & $N_{\mathrm{fs}}$ & Reference for age \\
\hline Alessi 13 & 8.72 & 104.34 & 48 & 10 & 3 & Yen et al. (2018) \\
Alessi 19 & 7.38 & 584.80 & 74 & 1 & 0 & Bossini et al. (2019) \\
Alessi 20 & 8.22 & 432.71 & 124 & 2 & 0 & WEBDA $^{a}$ \\
Alessi 21 & 7.816 & 581.40 & 137 & 2 & 0 & Bossini et al. (2019) \\
Alessi 24 & 7.945 & 485.67 & 163 & 1 & 0 & Bossini et al. (2019) \\
Alessi 3 & 8.90 & 280.43 & 178 & 8 & 0 & Yen et al. (2018) \\
Alessi 37 & 8.125 & 722.54 & 142 & 1 & 0 & Bossini et al. (2019) \\
Alessi 43 & 7.48 & 968.99 & 318 & 2 & 0 & WEBDA \\
Alessi 5 & 7.723 & 399.84 & 302 & 3 & 0 & Bossini et al. (2019) \\
Alessi 9 & 8.42 & 207.38 & 194 & 5 & 0 & Yen et al. (2018) \\
Alessi Teutsch 12 & 7.977 & 612.75 & 44 & 1 & 0 & Bossini et al. (2019) \\
Alessi Teutsch 5 & 7.02 & 900.09 & 158 & 1 & 0 & WEBDA \\
Alessi Teutsch 8 & 8.65 & 1034.13 & 341 & 2 & 0 & WEBDA \\
ASCC 105 & 7.994 & 560.85 & 127 & 1 & 0 & Bossini et al. (2019) \\
ASCC 10 & 8.599 & 685.40 & 71 & 1 & 0 & Bossini et al. (2019) \\
ASCC 12 & 8.42 & 1062.70 & 162 & 1 & 0 & WEBDA \\
ASCC 16 & 7.047 & 352.36 & 226 & 9 & 0 & Bossini et al. (2019) \\
ASCC 19 & 7.086 & 361.27 & 188 & 3 & 1 & Bossini et al. (2019) \\
ASCC 21 & 7.032 & 348.92 & 131 & 3 & 1 & Bossini et al. (2019) \\
ASCC 32 & 7.404 & 813.01 & 259 & 2 & 0 & Bossini et al. (2019) \\
ASCC 58 & 7.826 & 485.44 & 137 & 2 & 0 & Bossini et al. (2019) \\
ASCC 79 & 6.86 & 850.34 & 129 & 1 & 0 & Bossini et al. (2019) \\
ASCC 85 & 7.42 & 893.66 & 119 & 1 & 0 & WEBDA \\
Aveni Hunter 1 & 8.26 & 425.89 & 82 & 3 & 0 & WEBDA \\
Basel 8 & 8.102 & 1600.00 & 33 & 1 & 0 & WEBDA \\
Berkeley 86 & 7.116 & 1792.11 & 31 & 2 & 0 & WEBDA \\
Berkeley 87 & 7.152 & 1745.20 & 131 & 1 & 0 & WEBDA \\
BH 164 & 7.81 & 423.01 & 209 & 2 & 0 & Bossini et al. (2019) \\
BH 221 & 8.01 & 1131.22 & 162 & 1 & 0 & WEBDA \\
BH 23 & 7.14 & 443.07 & 94 & 1 & 0 & WEBDA \\
BH 56 & 7.24 & 927.64 & 122 & 3 & 0 & \\
\hline
\end{tabular}


Table 2. Continued.

\begin{tabular}{|c|c|c|c|c|c|c|}
\hline Name & $\log (\tau /[\mathrm{yr}])$ & $D[\mathrm{pc}]$ & $N_{\text {mem }}$ & $N_{\mathrm{lc}}$ & $N_{\mathrm{fs}}$ & Reference for age \\
\hline BH 99 & 7.908 & 449.44 & 389 & 1 & 0 & Bossini et al. (2019) \\
\hline Biurakan 2 & 7.011 & 1845.02 & 68 & 2 & 0 & WEBDA \\
\hline Blanco 1 & 7.975 & 237.53 & 381 & 82 & 12 & Bossini et al. (2019) \\
\hline Bochum 13 & 6.823 & 1763.67 & 73 & 1 & 0 & WEBDA \\
\hline Collinder 106 & 6.74 & 1574.80 & 114 & 1 & 0 & WEBDA \\
\hline Collinder 107 & 7.00 & 1620.75 & 159 & 1 & 0 & WEBDA \\
\hline Collinder 132 & 7.08 & 666.22 & 99 & 1 & 0 & WEBDA \\
\hline Collinder 135 & 7.407 & 305.06 & 352 & 1 & 0 & WEBDA \\
\hline Collinder 197 & 7.128 & 967.12 & 243 & 4 & 0 & WEBDA \\
\hline Collinder 258 & 7.834 & 1308.90 & 124 & 1 & 0 & Bossini et al. (2019) \\
\hline DBSB 104 & & 1101.32 & 27 & 1 & 0 & \\
\hline Dolidze 8 & & 1007.05 & 43 & 3 & 0 & \\
\hline FSR 0771 & 8.195 & 1618.12 & 48 & 1 & 0 & Bossini et al. (2019) \\
\hline FSR 0951 & 8.589 & 1808.32 & 195 & 1 & 0 & Bossini et al. (2019) \\
\hline Gulliver 11 & & 942.51 & 64 & 1 & 0 & \\
\hline Gulliver 21 & 8.472 & 664.89 & 126 & 1 & 0 & Bossini et al. (2019) \\
\hline Gulliver 6 & & 422.48 & 343 & 6 & 2 & \\
\hline Gulliver 9 & 7.154 & 503.78 & 265 & 1 & 0 & Bossini et al. (2019) \\
\hline Haffner 13 & 7.497 & 574.05 & 210 & 1 & 0 & Bossini et al. (2019) \\
\hline Harvard 10 & 7.899 & 705.22 & 164 & 1 & 0 & Bossini et al. (2019) \\
\hline Harvard 5 & 7.812 & 1308.90 & 52 & 1 & 0 & Bossini et al. (2019) \\
\hline IC 1396 & 7.054 & 938.09 & 460 & 2 & 0 & WEBDA \\
\hline IC 2391 & 7.561 & 151.93 & 224 & 7 & 0 & Bossini et al. (2019) \\
\hline IC 2395 & 7.223 & 725.16 & 297 & 4 & 0 & WEBDA \\
\hline IC 2488 & 8.2 & 1392.76 & 435 & 1 & 0 & Bossini et al. (2019) \\
\hline IC 2602 & 7.547 & 152.42 & 311 & 33 & 6 & Bossini et al. (2019) \\
\hline IC 2714 & 8.55 & 1390.82 & 934 & 1 & 0 & Bossini et al. (2019) \\
\hline IC 4651 & 9.057 & 946.97 & 854 & 8 & 0 & WEBDA \\
\hline IC 5146 & 6.00 & 824.40 & 108 & 2 & 0 & WEBDA \\
\hline King 6 & 8.582 & 742.94 & 234 & 1 & 0 & Bossini et al. (2019) \\
\hline L 1641S & & 437.06 & 101 & 1 & 0 & \\
\hline Mamajek 1 & 6.778 & 98.72 & 20 & 9 & 5 & WEBDA \\
\hline Mamajek 4 & 8.824 & 449.64 & 199 & 3 & 0 & Bossini et al. (2019) \\
\hline Melotte 111 & 8.652 & $96^{b}$ & 859 & 98 & 10 & WEBDA \\
\hline Melotte 20 & 7.938 & 176.43 & 764 & 50 & 0 & Bossini et al. (2019) \\
\hline Melotte 22 & 7.937 & 136.13 & 992 & 5 & 0 & Bossini et al. (2019) \\
\hline Muzzio 1 & & 1926.78 & 40 & 1 & 0 & \\
\hline
\end{tabular}


Table 2. Continued.

\begin{tabular}{|c|c|c|c|c|c|c|}
\hline Name & $\log (\tau /[\mathrm{yr}])$ & $D[\mathrm{pc}]$ & $N_{\text {mem }}$ & $N_{\mathrm{lc}}$ & $N_{\mathrm{fs}}$ & Reference for age \\
\hline NGC 129 & 7.886 & 1956.95 & 392 & 1 & 0 & WEBDA \\
\hline NGC 1333 & & 299.04 & 50 & 3 & 0 & \\
\hline NGC 1545 & 8.019 & 731.53 & 139 & 1 & 0 & Bossini et al. (2019) \\
\hline NGC 1579 & & 552.49 & 56 & 2 & 0 & \\
\hline NGC 1662 & 8.957 & 416.67 & 238 & 24 & 2 & Bossini et al. (2019) \\
\hline NGC 188 & 9.632 & 1972.39 & 864 & 3 & 0 & WEBDA \\
\hline NGC 1901 & 8.918 & 424.09 & 75 & 15 & 0 & Bossini et al. (2019) \\
\hline NGC 2232 & 7.87 & 326.05 & 198 & 2 & 0 & WEBDA \\
\hline NGC 2244 & 6.896 & 1620.75 & 623 & 13 & 0 & WEBDA \\
\hline NGC 2287 & 8.486 & 735.29 & 645 & 1 & 0 & Bossini et al. (2019) \\
\hline NGC 2323 & 7.975 & 1003.01 & 866 & 1 & 0 & Bossini et al. (2019) \\
\hline NGC 2354 & 8.126 & 1328.02 & 276 & 2 & 0 & WEBDA \\
\hline NGC 2422 & 8.104 & 483.09 & 442 & 43 & 1 & Bossini et al. (2019) \\
\hline NGC 2423 & 8.991 & 956.94 & 430 & 1 & 0 & Bossini et al. (2019) \\
\hline NGC 2437 & 8.390 & 1658.37 & 1797 & 1 & 0 & WEBDA \\
\hline NGC $2451 \mathrm{~A}$ & 7.647 & 193.61 & 337 & 26 & 1 & Bossini et al. (2019) \\
\hline NGC 2451B & 7.592 & 367.78 & 298 & 15 & 2 & Bossini et al. (2019) \\
\hline NGC 2516 & 8.4 & 413.74 & 798 & 144 & 1 & Bossini et al. (2019) \\
\hline NGC 2546 & 7.962 & 967.12 & 165 & 1 & 0 & Bossini et al. (2019) \\
\hline NGC 2547 & 7.432 & 391.70 & 233 & 4 & 0 & Bossini et al. (2019) \\
\hline NGC 2548 & 8.557 & 775.80 & 479 & 37 & 1 & WEBDA \\
\hline NGC 3114 & 8.093 & 1048.22 & 1296 & 6 & 0 & WEBDA \\
\hline NGC 3532 & 8.601 & 484.03 & 1889 & 11 & 0 & Bossini et al. (2019) \\
\hline NGC 3680 & 9.077 & 1071.81 & 100 & 8 & 0 & WEBDA \\
\hline NGC 5662 & 7.968 & 776.40 & 255 & 1 & 0 & WEBDA \\
\hline NGC 5822 & 8.95 & 842.46 & 667 & 1 & 0 & Bossini et al. (2019) \\
\hline NGC 6124 & 8.147 & 642.26 & 1327 & 1 & 0 & WEBDA \\
\hline NGC 6178 & 7.248 & 909.92 & 45 & 1 & 0 & WEBDA \\
\hline NGC 6193 & 6.775 & 1231.53 & 465 & 2 & 0 & WEBDA \\
\hline NGC 6231 & 6.843 & 1697.79 & 653 & 11 & 0 & WEBDA \\
\hline NGC 6242 & 7.767 & 1324.50 & 523 & 1 & 0 & Bossini et al. (2019) \\
\hline NGC 6250 & 7.415 & 1012.15 & 85 & 1 & 0 & WEBDA \\
\hline NGC 6281 & 8.497 & 533.90 & 513 & 6 & 0 & WEBDA \\
\hline NGC 6475 & 8.477 & 279.96 & 941 & 29 & 0 & Bossini et al. (2019) \\
\hline NGC 6811 & 8.936 & 1149.43 & 306 & 4 & 0 & Bossini et al. (2019) \\
\hline NGC 6866 & 8.89 & 1457.73 & 104 & 1 & 0 & Bossini et al. (2019) \\
\hline NGC 6871 & 6.958 & 1945.53 & 594 & 11 & 0 & WEBDA \\
\hline
\end{tabular}


Table 2. Continued.

\begin{tabular}{|c|c|c|c|c|c|c|}
\hline Name & $\log (\tau /[\mathrm{yr}])$ & $D[\mathrm{pc}]$ & $N_{\text {mem }}$ & $N_{\mathrm{lc}}$ & $N_{\mathrm{fs}}$ & Reference for age \\
\hline NGC 6910 & 7.127 & 1834.86 & 159 & 2 & 0 & WEBDA \\
\hline NGC 6913 & 7.111 & 1808.32 & 89 & 2 & 0 & WEBDA \\
\hline NGC 6940 & 9.01 & 1055.97 & 593 & 4 & 0 & Bossini et al. (2019) \\
\hline NGC 7092 & 8.491 & 299.67 & 161 & 6 & 0 & Bossini et al. (2019) \\
\hline NGC 7129 & & 920.81 & 36 & 1 & 0 & \\
\hline NGC 7243 & 8.006 & 896.06 & 313 & 2 & 0 & Bossini et al. (2019) \\
\hline NGC 743 & & 1135.07 & 62 & 1 & 0 & \\
\hline NGC 752 & 9.05 & 446.63 & 240 & 9 & 0 & WEBDA \\
\hline Pismis 5 & 7.197 & 974.66 & 92 & 1 & 0 & WEBDA \\
\hline Platais 3 & 8.319 & 177.94 & 90 & 12 & 0 & Bossini et al. (2019) \\
\hline Platais 8 & 7.75 & 134.86 & 211 & 13 & 3 & WEBDA \\
\hline Platais 9 & 7.894 & 183.05 & 128 & 18 & 4 & Bossini et al. (2019) \\
\hline Pozzo 1 & 7.117 & 350.51 & 390 & 3 & 0 & Bossini et al. (2019) \\
\hline Roslund 3 & 7.643 & 1709.40 & 112 & 1 & 0 & Bossini et al. (2019) \\
\hline RSG 5 & 7.70 & 339.56 & 173 & 1 & 0 & Röser et al. (2016) \\
\hline RSG 7 & 8.30 & 427.90 & 133 & 1 & 0 & Röser et al. (2016) \\
\hline RSG 8 & 8.50 & 452.49 & 211 & 1 & 0 & Röser et al. (2016) \\
\hline Ruprecht 91 & 6.90 & 1078.75 & 214 & 2 & 0 & WEBDA \\
\hline Stephenson 1 & 7.435 & 359.84 & 97 & 3 & 0 & Bossini et al. (2019) \\
\hline Stock 12 & 8.188 & 442.28 & 109 & 2 & 0 & Bossini et al. (2019) \\
\hline Stock 1 & 8.676 & 408.66 & 183 & 2 & 0 & Bossini et al. (2019) \\
\hline Stock 23 & 7.973 & 619.96 & 89 & 3 & 0 & Bossini et al. (2019) \\
\hline Stock 2 & 8.23 & 378.64 & 1190 & 1 & 0 & WEBDA \\
\hline Stock 5 & 7.73 & 987.17 & 115 & 1 & 0 & WEBDA \\
\hline Stock 7 & 7.214 & 693.96 & 141 & 1 & 0 & WEBDA \\
\hline Teutsch 38 & 8.14 & 665.78 & 109 & 1 & 0 & WEBDA \\
\hline Trumpler 10 & 7.74 & 437.25 & 478 & 2 & 0 & Bossini et al. (2019) \\
\hline Trumpler 2 & 7.962 & 698.81 & 179 & 2 & 0 & Bossini et al. (2019) \\
\hline Trumpler 3 & 7.83 & 684.46 & 222 & 2 & 0 & WEBDA \\
\hline Turner 5 & 8.49 & 421.41 & 28 & 1 & 0 & WEBDA \\
\hline vdBergh 1 & 8.025 & 1912.05 & 73 & 1 & 0 & WEBDA \\
\hline
\end{tabular}

${ }^{a}$ WEBDA database https://webda.physics.muni.cz/

${ }^{b}$ Distance for Melotte 111 are taken from WEBDA. 